\title{
Observed Propagation and Structure of the 33-h Atmospheric Kelvin Wave
}

\author{
ADRIAN J. MATTHEWS \\ Cooperative Research Centre for Southern Hemisphere Meteorology, Monash University, Melbourne, Australia \\ Roland A. MADDEN \\ NCAR, Boulder, Colorado
}

(Manuscript received 19 October 1999, in final form 2 March 2000)

\begin{abstract}
The structure of the 33-h Kelvin wave, a normal mode of the atmosphere, is examined in 6-hourly station and NCEP-NCAR reanalysis data. Cross-spectral analysis of 6 yr (1993-98) of tropical station pressure data shows a peak in coherence in a narrow frequency band centered near 0.74 cycles per day, corresponding to a period of approximately $33 \mathrm{~h}$. The phase angles are consistent with an eastward-propagating zonal-wavenumber-1 structure, implying an equatorial phase speed of approximately $340 \mathrm{~m} \mathrm{~s}^{-1}$. The global structure of the mode is revealed by empirical orthogonal function and regression analysis of $31 \mathrm{yr}$ (1968-98) of reanalysis data. The horizontal structure shows a zonal-wavenumber-1 equatorial Kelvin wave with an equatorial trapping scale of approximately $34^{\circ}$ lat. The vertical structure has zero phase change. The amplitude of the wave is approximately constant in the troposphere with an equatorial geopotential height perturbation of $0.9 \mathrm{~m}$, and then increases exponentially with height in the stratosphere. Cross-spectral analysis between the station and reanalysis data shows that the results from the two datasets are consistent. No evidence can be found for forcing of the wave by deep tropical convection, which is is examined using a twice-daily outgoing longwave radiation dataset.
\end{abstract}

\section{Introduction}

Theoretical treatments of the atmosphere predict an eastward-propagating gravity wave of zonal-wavenumber-1 longitudinal scale and a period near $33 \mathrm{~h}$ (Hough 1898; Haurwitz 1937; Longuet-Higgins 1968). It is an external (no phase change with height) equatorially trapped Kelvin wave that propagates with a phase speed of $c \approx 340 \mathrm{~m} \mathrm{~s}^{-1}$. Matsuno (1980) provided the first observational evidence for the wave in spectra of pressure data from Quito, Ecuador $\left(0.1^{\circ} \mathrm{S}, 78.5^{\circ} \mathrm{W}\right)$, and Luanda, Angola $\left(8.8^{\circ} \mathrm{S}, 13.2^{\circ} \mathrm{E}\right)$, which showed small relative maxima in variance with periods near 33 h. Cross-spectra between stations revealed coherence "not high enough to be taken significant," but a phase difference consistent with an eastward-propagating zonal-wavenumber-1 wave. Hamilton (1984) looked at 15$\mathrm{yr}$ records of 3-hourly pressure data at Kwajalein, Marshall Islands $\left(8.7^{\circ} \mathrm{N}, 167.7^{\circ} \mathrm{E}\right)$, Pohnpei, Caroline Islands $\left(7.0^{\circ} \mathrm{N}, 158.2^{\circ} \mathrm{E}\right)$, and Koror, Palau $\left(7.3^{\circ} \mathrm{N}\right.$, $134.5^{\circ} \mathrm{E}$ ), and found discernible power with periods of 32-33 h. He called the wave the "33-hour wave" and

Corresponding author address: Dr. Adrian Matthews, School of Environmental Sciences, University of East Anglia, Norwich NR4 7TJ, United Kingdom.

E-mail: a.j.matthews@uea.ac.uk estimated its amplitude to be $15 \mathrm{~Pa}$. In a follow-up paper Hamilton and Garcia (1986) found evidence of the 33-h wave in the spectrum of $49 \mathrm{yr}$ of hourly surface pressure data from Djarkata $\left(6.2^{\circ} \mathrm{S}, 106.8^{\circ} \mathrm{E}\right)$.

We recently began looking for the first-internal-mode Kelvin wave found over the Pacific (Milliff and Madden 1996) in 6-hourly sea level pressure data from seven stations in the equatorial Atlantic region and one in the Indian Ocean (see Fig. 1 for station locations; the western Pacific station of Chuuk was added later for a more global picture). That search continues, but a regular feature in our initial results relates to the $33-\mathrm{h}$ wave and is reported on here. Using cross-spectra between stations, we add to Matsuno's evidence that the spectral "peaks" reported by him, Hamilton, and Garcia do indeed reflect an eastward-propagating zonal wavenumber 1 wave. In addition, empirical orthogonal function and regression analysis were used to extract the three-dimensional structure of the wave from 6-hourly National Centers for Environmental Prediction-National Center for Atmospheric Research (NCEP-NCAR) reanalyses.

\section{Theoretical background}

The theoretical structure of the Kelvin wave normal mode is a solution of the primitive equations linearized about a resting isothermal atmosphere (e.g., Andrews 


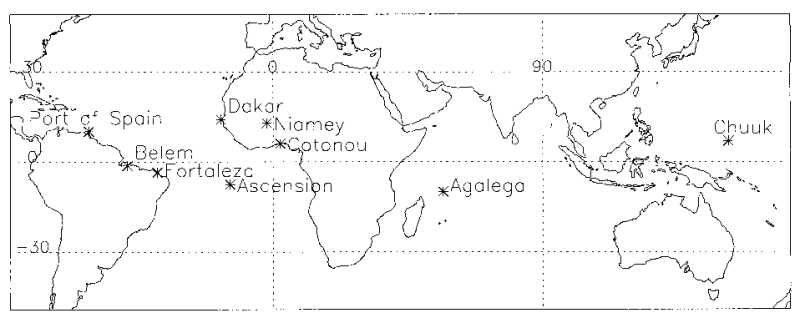

FIG. 1. The locations of the nine stations in Table 1.

et al. 1987). A solution that is separable into vertical and horizontal parts is sought. With no forcing, the vertical structure of the wind and geopotential height anomalies is that of the external mode and proportional to $\exp (\kappa z / H)$, where $z$ is height; $\kappa=R / c_{p}=2 / 7$ is the ratio of the gas constant $R$ to the specific heat at constant pressure $c_{p}$; and $H=R T_{s} / g$ is the density scale height, where $T_{s}$ is the global mean temperature and $g$ is the acceleration due to gravity. Although the amplitude of the anomalies increases with height, the energy density decreases with height as the air density profile has the form $\exp (-z / H)$. In pressure coordinates, the vertical structure has the form $\left(p / p_{s}\right)^{-\kappa}$, where $p_{s}=1000 \mathrm{hPa}$ is a reference pressure level.

The horizontal and time dependence of the mode is governed by Laplace's tidal equations and has been documented by Longuet-Higgins (1968). However, if the equatorial $\beta$-plane approximation of Cartesian geometry is made, then, following Matsuno (1966), the structure of the Kelvin wave has the simple analytical form

$$
\begin{aligned}
& u=u_{0}\left(\frac{p}{p_{s}}\right)^{-\kappa} e^{-\beta y^{2} / 2 c} e^{i k(x-c t)} \\
& v=0 \\
& Z=\frac{c}{g} u_{0}\left(\frac{p}{p_{s}}\right)^{-\kappa} e^{-\beta y^{2} / 2 c} e^{i k(x-c t),}
\end{aligned}
$$

where $u$ and $v$ are the zonal and meridional wind perturbations, $Z$ is the geopotential height perturbation, $u_{0}$ is the maximum amplitude of the equatorial zonal wind anomaly at the reference pressure level $p_{s}$, and $\beta=2.3$ $\times 10^{-11} \mathrm{~m}^{-1} \mathrm{~s}^{-1}$ is the planetary vorticity gradient at the equator. The phase speed of the wave is $c=(g h)^{1 / 2}$, where the equivalent depth $h=\gamma H$ and $\gamma=c_{p} / c_{v}=$ $7 / 5$ is the ratio of the specific heat at constant pressure to the specific heat at constant volume. For a global mean temperature of $T_{s}=283 \mathrm{~K}$, the scale height is $H$ $=8.3 \mathrm{~km}$, the equivalent depth is $h=11.6 \mathrm{~km}$, and the phase speed is $c=340 \mathrm{~m} \mathrm{~s}^{-1}$.

The perturbations follow a Gaussian distribution about the equator with a trapping scale of

$$
y_{0}=(c / \beta)^{1 / 2} \approx 3800 \mathrm{~km} \approx 34^{\circ} \text { lat. }
$$

The fractional error in computing $f$ by making the equatorial $\beta$-plane approximation is $6 \%$ at this latitude. More accurate calculations with a realistic temperature profile give an equivalent depth close to $10 \mathrm{~km}$ (Salby 1979). In addition, if the full spherical geometry is taken into account, the equatorial phase speed is then $342 \mathrm{~m} \mathrm{~s}^{-1}$, giving a period of $32.5 \mathrm{~h}$ (Hamilton and Garcia 1986). It should be noted that the assumption of a resting or motionless atmosphere is a very accurate approximation, as the phase speed of the Kelvin wave $\left(340 \mathrm{~m} \mathrm{~s}^{-1}\right)$ is much larger than typical tropical mean wind speeds (up to $20 \mathrm{~m} \mathrm{~s}^{-1}$ ).

\section{Data}

Table 1 contains information about the nine stations whose sea level pressures we have studied. The period of record is for $6 \mathrm{yr}$ (2191 days) from 1 January 1993 to 31 December 1998. Nominally four observations (0000, 0600, 1200, 1800 UTC) were available per day. Missing data indicated in Table 1 were replaced by linear interpolation in time. In addition, any outlier data that differed from the long-term average by more than 3 standard deviations was replaced by linear interpolation. The total number of interpolated values are the sum of the missing and outlier columns from Table 1.

The global structure of the 33-h Kelvin wave was examined with the 6-hourly NCEP-NCAR reanalysis data over the 31-yr period from 1 January 1968 to 31 December 1998 (Kalnay et al. 1996). A possible relationship with deep tropical convection was examined using a global, gridded, interpolated satellite outgoing

\begin{tabular}{|c|c|c|c|c|c|c|}
\hline Station & Latitude & Longitude & Missing & Outliers & Mean $(\mathrm{hPa})$ & $\begin{array}{c}\text { Variance } \\
(\mathrm{hPa})^{2}\end{array}$ \\
\hline Port of Spain & $10.6^{\circ} \mathrm{N}$ & $61.4^{\circ} \mathrm{W}$ & 421 & 46 & 1013.2 & 1.6 \\
\hline Belem & $1.4^{\circ} \mathrm{S}$ & $48.5^{\circ} \mathrm{W}$ & 1023 & 18 & 1011.4 & 1.5 \\
\hline Fortaleza & $3.8^{\circ} \mathrm{S}$ & $38.5^{\circ} \mathrm{W}$ & 1279 & 22 & 1012.7 & 1.6 \\
\hline Dakar & $14.7^{\circ} \mathrm{N}$ & $17.5^{\circ} \mathrm{W}$ & 214 & 21 & 1012.4 & 2.2 \\
\hline Ascension & $8.0^{\circ} \mathrm{S}$ & $14.4^{\circ} \mathrm{W}$ & 390 & 49 & 1014.1 & 1.3 \\
\hline Niamey & $13.5^{\circ} \mathrm{N}$ & $2.2^{\circ} \mathrm{W}$ & 530 & 13 & 1009.9 & 3.6 \\
\hline Cotonou & $6.4^{\circ} \mathrm{N}$ & $2.4^{\circ} \mathrm{E}$ & 663 & 9 & 1010.9 & 1.4 \\
\hline Agalega & $10.4^{\circ} \mathrm{S}$ & $56.8^{\circ} \mathrm{E}$ & 711 & 18 & 1012.4 & 2.0 \\
\hline Chuuk & $7.3^{\circ} \mathrm{N}$ & $151.8^{\circ} \mathrm{E}$ & 941 & 32 & 1009.8 & 2.6 \\
\hline
\end{tabular}
longwave radiation (OLR) dataset. Two versions of the OLR data were available: a twice-daily dataset over the

TABLE 1. Information on station data. 

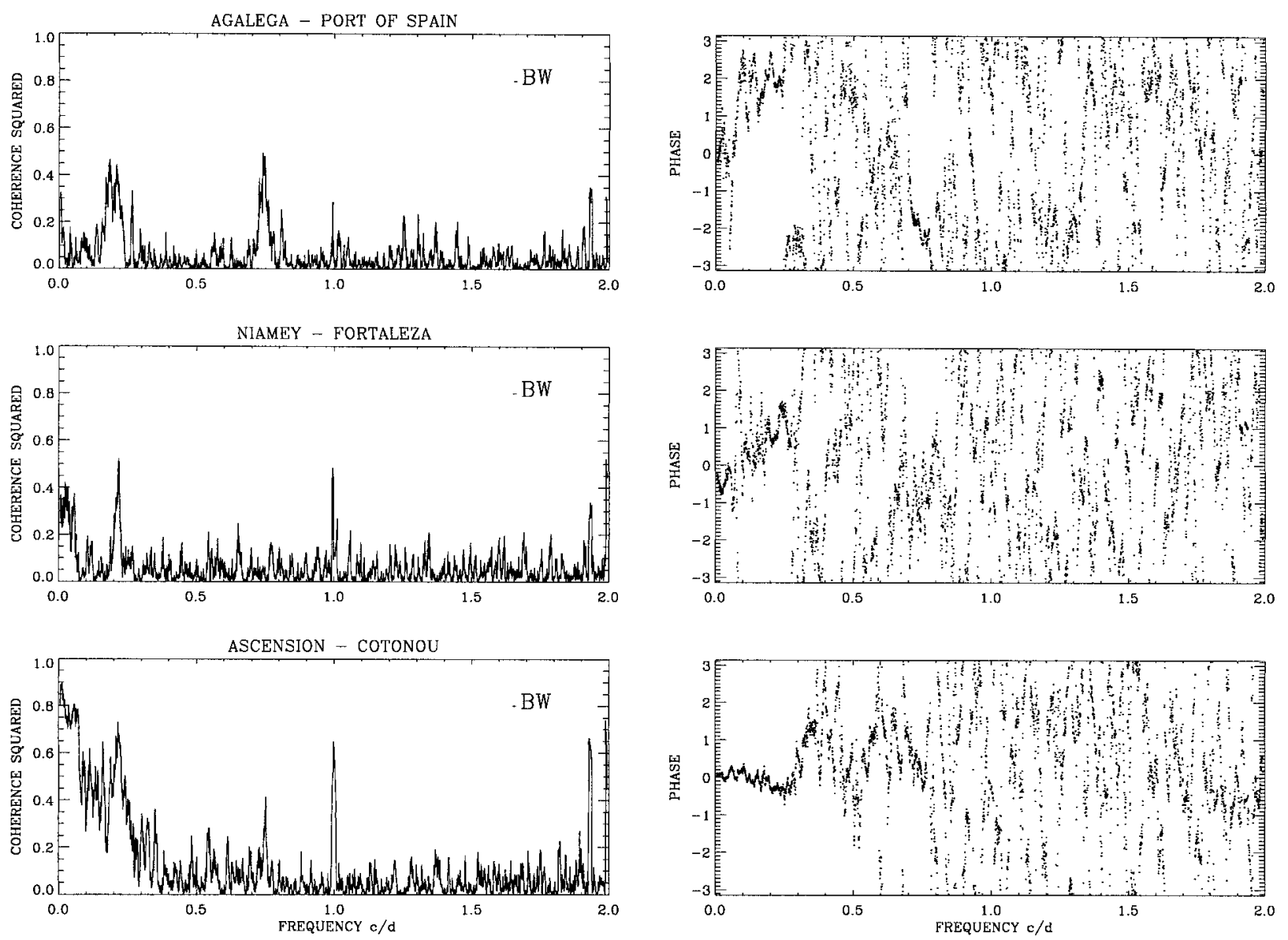

FIG. 2. Coherence squared (left panels) and phase angles (right panels) for cross-spectra between Agalega and Port of Spain (top), Niamey and Fortaleza (middle), and Ascension and Cotonou (bottom). The narrow bandwidth (BW) of $0.0114 \mathrm{cpd}$ is indicated. Phases angles are in radians.

period 1 January 1993-9 May 1998 (Wheeler and Kiladis 1999), and a daily-mean dataset over the period 1 June 1974-16 September 1998, excluding the period 16 March 1978-31 December 1978 (Liebmann and Smith 1996). Areas of deep tropical convection are associated with high, cold cloud tops and low values of OLR.

\section{Station data}

\section{a. Cross-spectral analysis}

Spectra were estimated by Fourier transforming the $8764(4 \times 2191)$ points in the time series and squaring and summing the resulting coefficients to form the periodogram. Next, a running average of 25 periodogram estimates gave a spectrum with frequency resolution of 0.0029 cycles per $6 \mathrm{~h}$ or 0.0114 cycles per day (cpd) and approximately $50(25 \times 2)$ degrees of freedom $(\mathrm{df})$. Cross spectra were estimated by multiplication of the real and imaginary parts of the Fourier transform of two time series, computing a running average of the resulting products to give the cospectrum and quadrature spectrum, and, in turn, phase and coherence squared.
The seasonal variation in the mean of the data was removed by subtracting the annual, semiannual, and triannual components. Diurnal and semidiurnal components were also removed. This was done by Fourier transforming the data, setting the appropriate coefficients to zero, and then retransforming. The periodogram of the resulting data has zero values at the five harmonics that are removed. Because of the 25-point running average, these zero values have little effect on the final spectrum; nevertheless, periodogram values at the two harmonics nearest the annual and semiannual frequency, where variance is likely to be relatively large, were replaced by the average of adjacent values before the running average procedure. The time series data were Fourier transformed after a cosine taper was applied to $5 \%$ of the values at each end. The variance values in Table 1 were computed after the seasonal cycle was removed but before the data were tapered.

\section{b. Results}

Time series from the nine stations resulted in 36 separate cross-spectra. Figure 2 shows the resulting coher- 


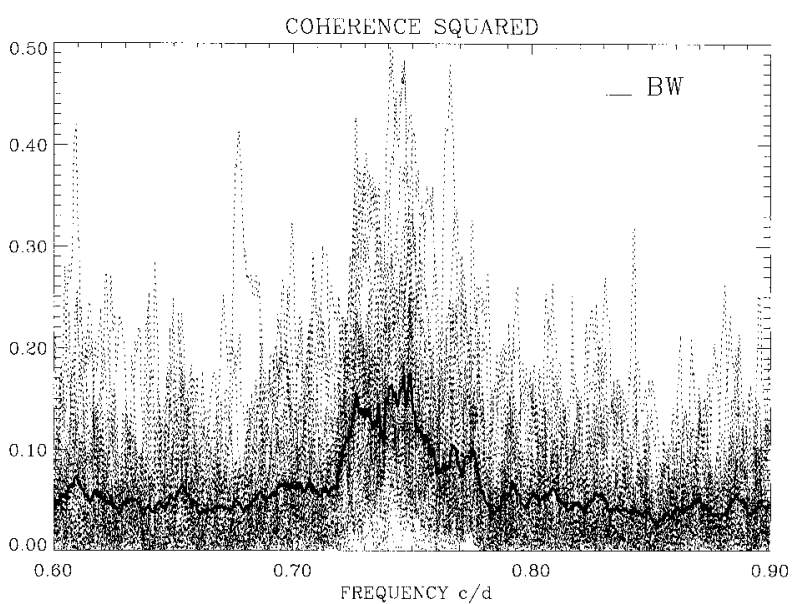

FIG. 3. Coherence squares in the 0.6-0.9-cpd frequency range from all 36 possible cross-spectra between the eight stations are plotted with dotted lines. The arithmetic mean is shown by the heavy line. The line to the left of BW indicates the bandwidth of the analysis.

ence squares and phase angles for three of them. Large coherence near 0.2 cpd corresponds to the 5-day Rossby-Haurwitz wave (Madden and Julian 1972). A positive phase angle means that the first named station leads the second. Hence, Agalega leads Port of Spain, Niamey leads Fortaleza, and Ascension trails Cotonou at 5-day periods, all indicating westward propagation.

There are large coherence squares evident in all three cross-spectra near 24-h (about $0.996 \mathrm{cpd}$ ) and 12-h (1.93 and $1.99 \mathrm{cpd}$ ) periods. The variation at $1.93 \mathrm{cpd}$ is the M2 tide identified by Matsuno (1980). It is likely that the other large coherences are manifestations of solar tides at 24 and $12 \mathrm{~h}$ that have survived the filtering.

There is large coherence squared near the 33-h period (0.727 cpd) between Agalega and Port of Spain and also between Ascension and Cotonou. Phase angles indicate that Agalega trails Port of Spain and Ascension leads Cotonou, both characteristic of the eastward propagation expected for an equatorially trapped Kelvin wave. Phase angles indicate that Niamey trails Fortaleza near the 33-h period, again as expected for an eastward-moving Kelvin wave, despite the fact that coherence squared between these two stations is low. This is similar to the cross-spectral results between Quito and Luanda reported by Matsuno (1980); the phase angles were correct but coherence was small.

All 36 coherence squares in the 0.6-0.9-cpd frequency range are plotted with dotted lines and their arithmetic average is indicated by the heavy line in Fig. 3 . If there are $50(25 \times 2)$ df for a spectral estimate, then the $95 \%$ confidence limit for a null hypothesis of zero coherence squared is 0.12 . Many of the coherence squares do exceed that value, and possibly more telling are the relatively high average values and the fact that there are very few individual values near zero in the $0.72-0.76-$ cpd $(33.3-31.6 \mathrm{~h})$ range. According to the

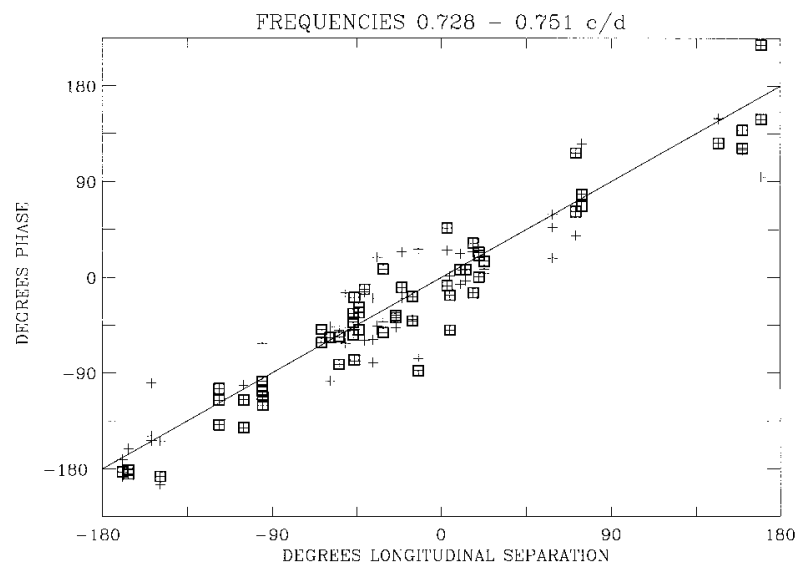

FIG. 4. Phase angles (ordinate) from the 36 cross-spectra plotted against longitudinal distance between the two corresponding stations (abscissa). There are phase angles at $0.7284,0.7398$, and $0.7513 \mathrm{cpd}$ plotted for each cross-spectrum. Phase angles whose coherence squared exceeds 0.12 are indicated by boxed crosses. Negative phase was chosen to signify that the pressure of the station to the west leads that of the station to the east. Accordingly, phases that lie on the diagonal line are consistent with an eastward-propagating zonalwavenumber-1 disturbance.

band width depicted in the upper right-hand corner, there are about three independent estimates in that range.

In order to establish the longitudinal scale and possible phase propagation of the disturbance responsible for the apparent coherence in the 0.72-0.76-cpd range, phase angles between stations were plotted as a function of their longitudinal separation (Fig. 4). Phase angles estimated at $0.7284,0.7398$, and $0.7513 \mathrm{cpd}$ are included. Phase angles for an eastward-propagating zonalwavenumber-1 wave would fall along the diagonal line from lower left to upper right. Values whose coherence squared are significantly different from zero $(>0.12)$ are indicated by the boxed crosses. Many coherences are not statistically significant but nearly all phase angles fall close to the diagonal. Estimated phase angles with coherence squared of 0.12 have $95 \%$ confidence limits of $\pm 30^{\circ}$ (Jenkins and Watts 1968, p. 381). Most of the "significant" phases fall within these limits about the eastward, wave-1 diagonal.

Figure 5 shows the nine individual spectra along with their arithmetic average. There is a relative maximum in the 0.72-0.76-cpd range, but it probably (we do not know the df of the average spectrum for certain) is not significantly different from a white noise spectrum. We can get a first-order estimate of the amplitude of the 33-h wave from the average spectrum and coherence of Figs. 5 and 3, respectively. The average spectral value between 0.72 and $0.76 \mathrm{cpd}$ (from Fig. 5) is about 0.85 $\mathrm{hPa}^{2} \mathrm{cpd}^{-1}$. The variance in the band is then $\left(0.85 \mathrm{hPa}^{2}\right.$ $\left.\mathrm{cpd}^{-1}\right) \times((0.76-0.72) \mathrm{cpd})=0.034 \mathrm{hPa}^{2}$. Since the average coherence squared in the band is approximately 0.15 (Fig. 3), we will assume that $15 \%$ of the total variance in the band is associated with the wave, or $0.0051 \mathrm{hPa}^{2}$. The variance of a sinusoid with amplitude 


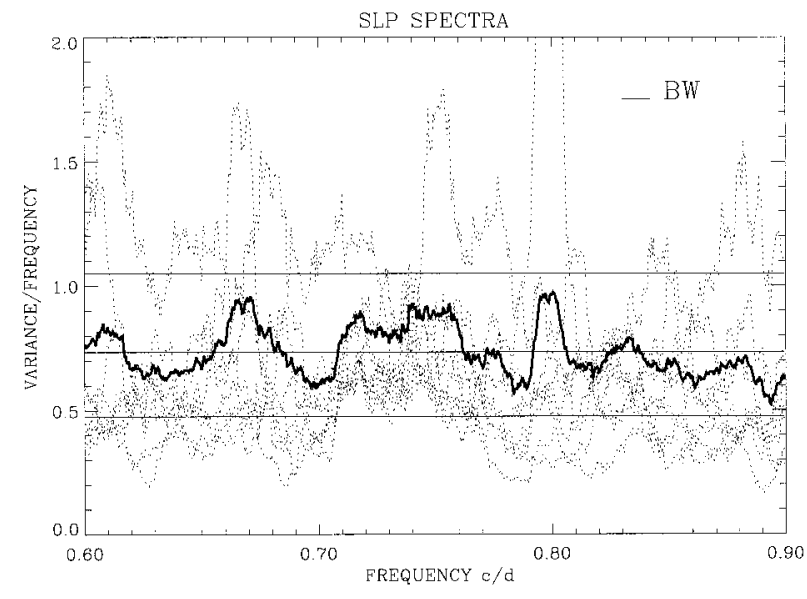

FIG. 5. Spectral estimates in the 0.6-0.9-cpd frequency range for the pressures at the nine stations are plotted with dotted lines. The arithmetic mean is shown by the heavy line. Thin horizontal lines represent a white noise null hypothesis and the $95 \%$ confidence limits assuming $50 \mathrm{df}$. The values on the ordinate are in $\mathrm{hPa}^{2} \mathrm{cpd}^{-1}$.

$A$ is $A^{2} / 2$, giving an amplitude of $A \approx 10 \mathrm{~Pa}$. This compares well with Hamilton's (1984) estimate of $A \approx$ $15 \mathrm{~Pa}$ when one considers that he integrated over a frequency band of $0.096 \mathrm{cpd}$, which is more than twice as large as the band used here.

\section{Global structure}

\section{a. Analysis technique}

The global structure of the 33-h Kelvin wave is now examined using $31 \mathrm{yr}$ of 6-hourly gridded NCEPNCAR reanalysis data. The cross-spectral analysis of the station data in section 4 shows high coherence squares associated with an eastward-propagating zonalwavenumber- 1 equatorial wave in the frequency range $0.72-0.76 \mathrm{cpd}$. Hence, the reanalysis data were first passed through a 241-weight bandpass Lanczos filter with half-amplitude points at 0.69 and $0.79 \mathrm{cpd}$ and near-unit response over the frequency range of interest (Fig. 6).

The global structure of the 33-h Kelvin wave was then determined from an empirical orthogonal function (EOF) analysis of the bandpass-filtered sea level pressure (SLP) reanalysis maps over the tropical domain $25^{\circ} \mathrm{S}-25^{\circ} \mathrm{N}$. The EOFs were defined as the leading eigenvectors of the SLP covariance matrix and were scaled so that their amplitude was equal to the square root of their eigenvalue. The principal component (PC) time series show the temporal amplitude of the EOFs and were calculated by projecting the EOF spatial structures onto the filtered SLP maps at each time. The PC time series were then rescaled to have unit variance by dividing by their eigenvalue. A comprehensive discussion of EOF analysis is given by Wilks (1995).

The structure of the 33-h Kelvin wave in any other variable (e.g., 500-hPa geopotential height) was deter-

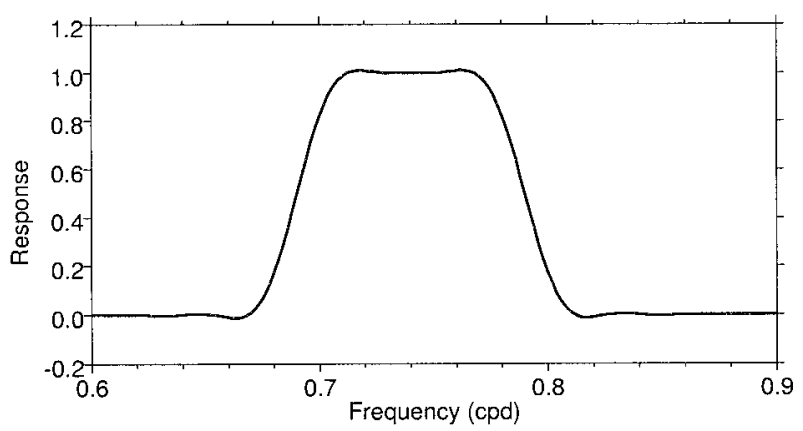

FIG. 6. Response function of the bandpass filter applied to the reanalysis data.

mined by linear regression with the PC time series. At each grid point $i$ a linear regression equation,

$$
\hat{y}_{i}=a_{i}+b_{i} x,
$$

was formed where $\hat{y}$ is the regressed value of the dependent variable $y$ (in this case the gridpoint time series of $500-\mathrm{hPa}$ geopotential height), $x$ is the independent variable (the time series of PC1), and $a_{i}$ and $b_{i}$ are constants to be determined for that particular grid point. A map of $\hat{y}$ is then plotted for a fixed value of $x$. The choice of the value of $x$ is rather arbitrary. However, if the PC time series varies approximately sinusoidally with amplitude $A$, then its variance will be $A^{2} / 2$. As the PC time series was scaled to have unit variance, then $A=\sqrt{2}$. Hence the regression maps are plotted for a fixed value of $x$, or PC1 $=\sqrt{2}$.

\section{b. Results}

\section{1) Sea level PRESSURe}

The first two EOFs of the bandpass-filtered SLP reanalysis data accounted for $\lambda_{1}=11.66 \%$ and $\lambda_{2}=$ $11.54 \%$ of the variance, respectively, where $\lambda_{j}$ are the eigenvalues. EOF3 only accounted for $\lambda_{3}=1.85 \%$ of the variance; therefore, we consider further only EOFs 1 and 2. EOF1 has a zonal wavenumber 1 structure with an equatorial maximum at $25^{\circ} \mathrm{W}$ and a minimum at $155^{\circ} \mathrm{E}$ (Fig. 7a). EOF2 also has a zonal wavenumber 1 structure, but shifted $90^{\circ}$ eastward (Fig. 7b). Figure 7c shows a representative sample of the PC time series for 10 days starting on 16 January 1994. The two time series are in quadrature with $\mathrm{PC} 1$ leading $\mathrm{PC} 2$ by a quarter cycle. Together with the spatial structures of EOFs 1 and 2, they describe an eastward-propagating equatorially trapped mode, consistent with an equatorial Kelvin wave.

The expected theoretical structure of the normal mode is a sinusoidal wave that moves eastward uniformly around the equator. Such a wave would be represented by a pair of leading EOFs with identical spatial structures, but shifted by $90^{\circ}$ longitude, and with equal eigenvalues $\lambda_{1}=\lambda_{2}$. The actual longitudinal positions of the maximum/minimum in EOF1, and therefore EOF2, 
(a) EOF 1

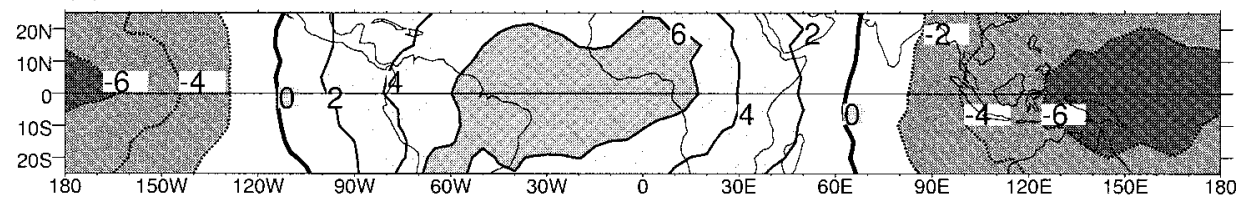

(b) EOF 2

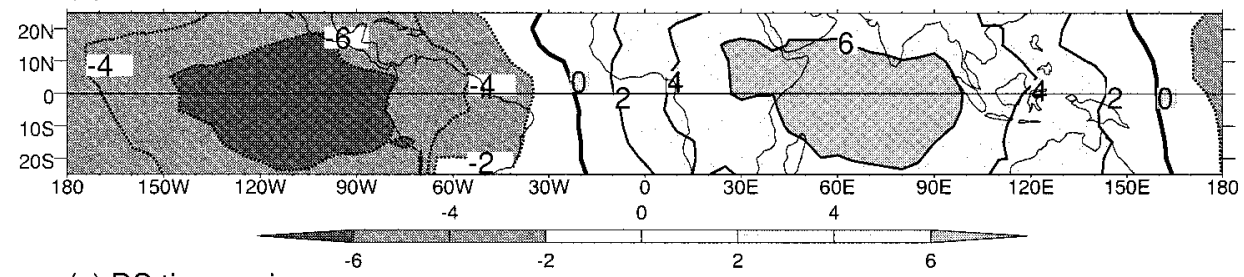

(c) PC time series

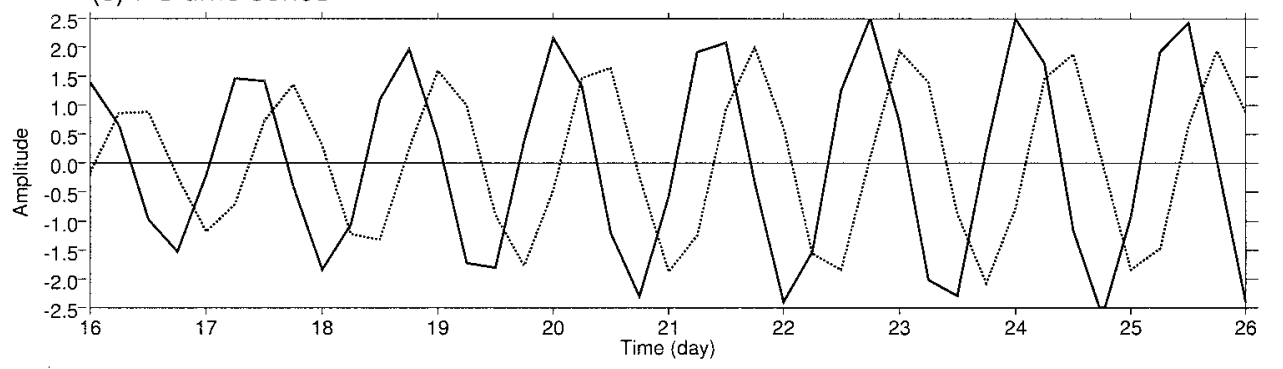

FIG. 7. EOF analysis of bandpass-filtered SLP reanalysis data. (a) EOF1, (b) EOF2. Contour interval is 2 $\mathrm{Pa}$; negative contours are dotted and the zero contour is thickened; shading is shown by the legend. (c) PC time series for 10 days starting on 16 Jan 1994. PC1 (PC2) is shown by the solid (dotted) line.

would be sample dependent. This degeneracy of EOFs 1 and 2 can be tested.

First, the degrees of freedom is estimated. The length of the filtered dataset is 11263 days with data points every $6 \mathrm{~h}$. Assuming the data are independent, there are $45052=4 \times 11263 \mathrm{df}$ distributed evenly between 0.0 and 0.5 cycles per $6 \mathrm{~h}$, and the filter passes Fourier components between 0.1725 and 0.1975 cycles per $6 \mathrm{~h}$ $(0.69$ and $0.79 \mathrm{cpd})$; then there are approximately $[(0.1975-0.1725) / 0.5] \times 45052=2250 \mathrm{df}$.

If the eigenvalues $\lambda$ of two adjacent eigenvectors are separated by less than $d \lambda=\lambda(2 / \mathrm{df})^{1 / 2}$, they are susceptible to sampling errors and are effectively degenerate (North et al. 1982). For EOF1, $\lambda_{1}=11.66 \%$ and $d \lambda=0.35 \%$ (this is certainly an underestimate of $d \lambda$ because the estimate of df is for independent data), which is greater than the difference $\lambda_{1}-\lambda_{2}=0.12 \%$. Hence EOFs 1 and 2 form a degenerate pair, consistent with the model of a uniformly propagating wave.

Regression maps of SLP with respect to PCs 1 and 2 (not shown) show similar patterns to EOFs 1 and 2, scaled by a factor of $\sqrt{2}$. As the equatorial amplitude of the EOF structures is approximately $7 \mathrm{~Pa}$, this gives a value of $A \approx 10 \mathrm{~Pa}$ for the amplitude of the 33-h Kelvin wave in the NCEP-NCAR reanalysis data, in agreement with the value of $A \approx 10 \mathrm{~Pa}$ obtained from the station data in section 4 .

\section{2) Geopotential height}

Figure 8 shows a set of regression maps with respect to PC1 of geopotential height at selected levels. All the maps show zonal-wavenumber-1 structures with equatorial maxima, similar to the SLP equatorial Kelvin wave structure in EOF1 (Fig. 7a). The amplitude of the geopotential height anomaly at $1000 \mathrm{hPa}$ is approximately $0.9 \mathrm{~m}$, consistent with the SLP anomaly of 10 $\mathrm{Pa}$ through the hydrostatic relationship.

The Kelvin wave appears to be in phase at all levels. This lack of vertical tilt is confirmed in a vertical section along the equator (Fig. 9) and is consistent with an external-mode vertical structure. Regression maps calculated with respect to PC2 (not shown) have a similar structure but are shifted $90^{\circ}$ to the east. The correlation map for the 1000-hPa geopotential height field (not shown) contains values in excess of 0.6 at the center of the equatorial anomalies and is highly significant, as the $5 \%$ significance level for $2250 \mathrm{df}$ is 0.04 .

The vertical and horizontal structure of the observed geopotential height anomalies can be compared with theoretical expectations [Eq. (3)]. The observed structure of the normal mode has a dominant zonal-wavenumber-1 component. The amplitude of this zonalwavenumber-1 component at the equator (averaged from $10^{\circ} \mathrm{S}$ to $10^{\circ} \mathrm{N}$ ) as a function of pressure is shown in Fig. 10; both axes are plotted on a logarithmic scale. 
(a) $10 \mathrm{hPa}$

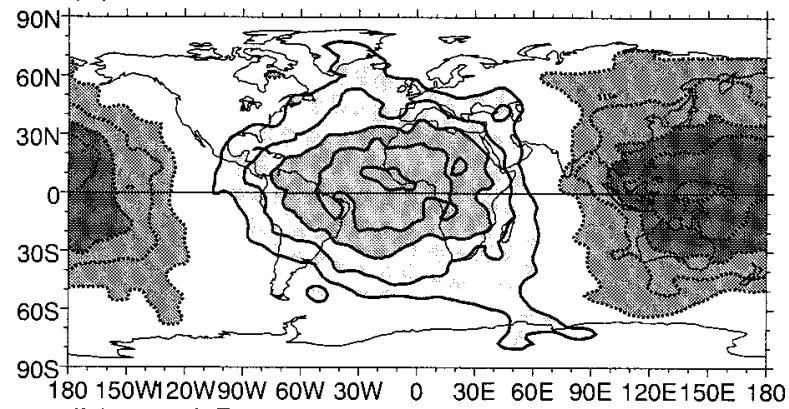

(b) $100 \mathrm{hPa}$

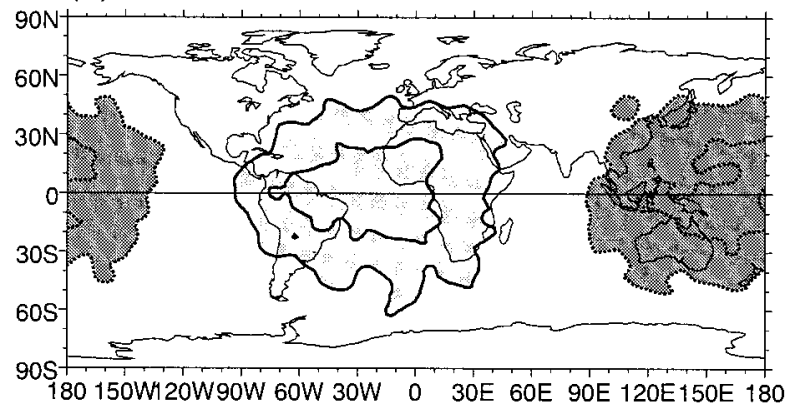

(c) $500 \mathrm{hPa}$

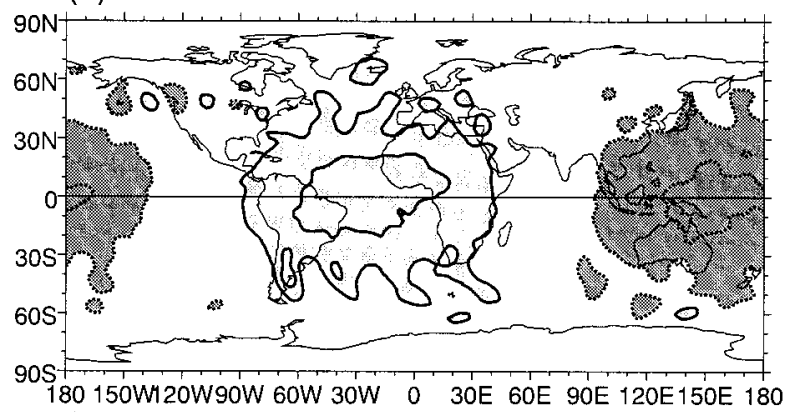

(d) $1000 \mathrm{hPa}$

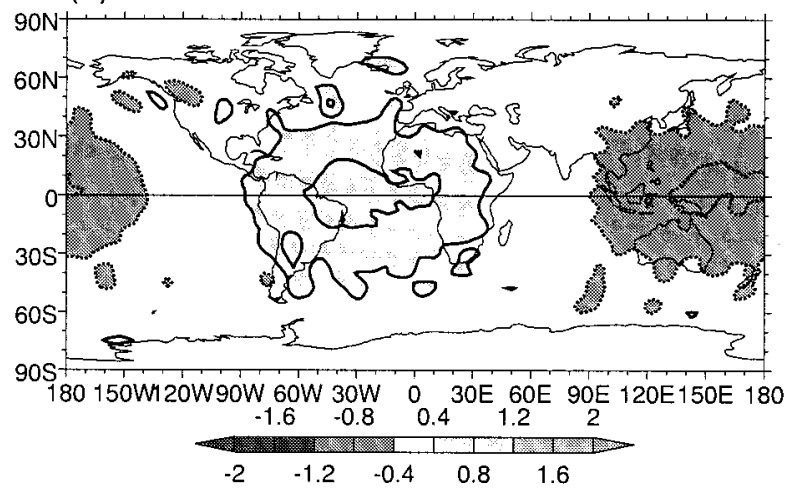

FIG. 8. Regression maps of geopotential height anomalies calculated with respect to PC1 of bandpass-filtered SLP and plotted for a fixed value of PC1 $=\sqrt{2}$, at (a) 10, (b) 100, (c) 500, and (d) 1000 $\mathrm{hPa}$. Contour interval is $0.4 \mathrm{~m}$; negative contours are dotted and the zero contour is omitted. Shading is shown by the legend.

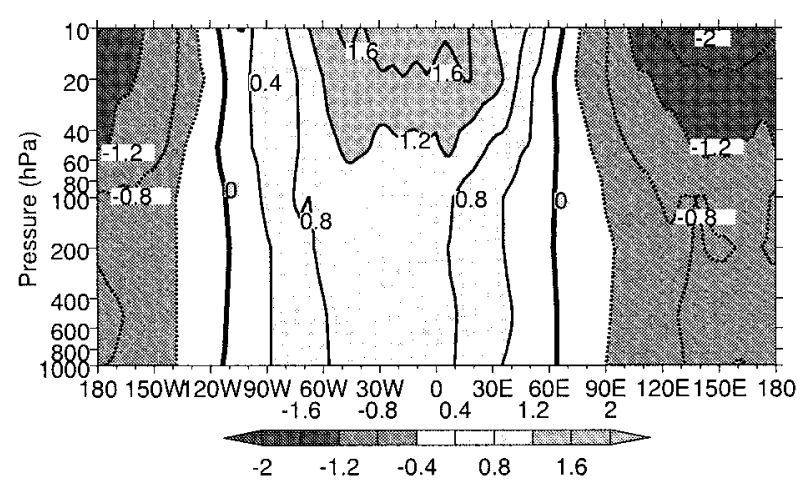

FIG. 9. As in Fig. 8 but for a vertical section of geopotential height anomalies along the equator (units are in meters).

The amplitude of the observed wave is approximately constant in the troposphere. On a log-log plot, the amplitude of the theoretical external mode follows a straight line with slope $\kappa$. The dotted line in Fig. 10 shows such an external mode and is a reasonable fit to the observed wave in the stratosphere.

The solid line in Fig. 11 shows a latitudinal section of the zonal-wavenumber- 1 component of the $500-\mathrm{hPa}$ geopotential height anomalies at $25^{\circ} \mathrm{W}$, the longitude of maximum SLP anomaly in EOF1 (Fig. 7a). The observations agree well with the theoretical Gaussian structure of an equatorial Kelvin wave with a phase speed of $340 \mathrm{~m} \mathrm{~s}^{-1}$ and a corresponding equatorial trapping scale of $34^{\circ}$ lat [Eq. (4)] shown by the dotted line, especially in the Tropics.

\section{3) ZONAL WIND}

An equatorial Kelvin wave has a zonal wind field with the same spatial structure as the geopotential height field [Eqs. (1) and (3); $u=(g / c) Z$ ]. For the external mode with its large phase speed, the factor $g / c \approx 0.029$ $\mathrm{s}^{-1}$ is very small. At $10 \mathrm{hPa}$ the zonal-wavenumber-1 component of the geopotential height anomaly in Fig. $8 \mathrm{a}$ has an amplitude of $1.9 \mathrm{~m}$; therefore the corresponding 10-hPa zonal-wavenumber-1 component of the zonal wind anomaly would be expected to have an amplitude of $0.055 \mathrm{~m} \mathrm{~s}^{-1}$. This is much less than the measured accuracy of the zonal wind field but may still be detectable in the statistical analysis used here.

The regression map of $10-\mathrm{hPa}$ zonal wind is shown in Fig. 12. It does have a prominent zonal-wavenumber-1 component but the signal-to-noise ratio is lower than in the geopotential height map of Fig. 8a. The amplitude of the zonal-wavenumber- 1 component of the $10-\mathrm{hPa}$ zonal wind anomaly is $0.055 \mathrm{~m} \mathrm{~s}^{-1}$, in agreement with the expected value.

4) TEMPORAL VARIABILITY AND POSSIBLE FORCING BY TROPICAL CONVECTION

The amplitude $A$ of the 33 -h Kelvin wave can be defined as 


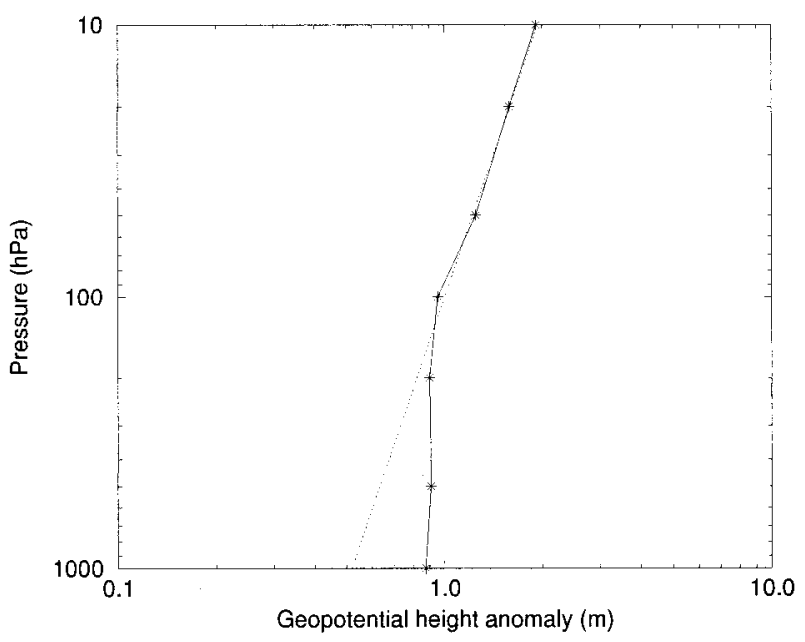

FIG. 10. Amplitude of the zonal-wavenumber-1 component of the regressed geopotential height anomalies averaged over $10^{\circ} \mathrm{S}-10^{\circ} \mathrm{N}$ (solid line). The theoretical structure of an external mode with a surface amplitude scaled to match the observations in the stratosphere is shown by the dotted line. Both axes are plotted on a logarithmic scale.

$$
A(t)=\left[\mathrm{PC}^{2}(t)+\mathrm{PC}^{2}(t)\right]^{1 / 2}
$$

This amplitude is calculated for the whole 30-yr time domain and is shown for the 5 yr from 1985 to 1990 in Fig. 13. The Kelvin wave is always present and it exhibits variability on many timescales, but its power spectrum (not shown) shows a red noise behavior, with no significant peaks at any frequency and, in particular, no annual cycle.

Deep tropical convection has been suggested as a possible mechanism for the 33-h Kelvin wave (Hamilton and Garcia 1986). Although the midtropospheric heating maximum associated with tropical convection tends to force structures with a first-internal-mode vertical structure most effectively, there may still be a small-amplitude projection of the heating onto the external-mode vertical structure of the 33-h Kelvin wave.

A direct relationship between the Kelvin wave and tropical convection (i.e., a disturbance in the tropical convection propagating eastward at $340 \mathrm{~m} \mathrm{~s}^{-1}$ ) would not be expected. However, there could be a forcing of the Kelvin wave by local convection amplifying and decaying in situ with a period of $33 \mathrm{~h}$. Hence, a regression map (not shown) with respect to PC1 was constructed from the twice-daily OLR data described in section 3. No coherent OLR signal was found.

It would be more reasonable to expect an indirect relationship between the Kelvin wave and tropical convection, with enhanced convection associated with the annual cycle or recognized low-frequency phenomena such as the Madden-Julian oscillation (MJO) leading to enhanced 33-h Kelvin wave activity. The zonal-wavenumber-1 component of the low-frequency convection would be expected to force the Kelvin wave most effectively. Hence, a time series of the amplitude of the

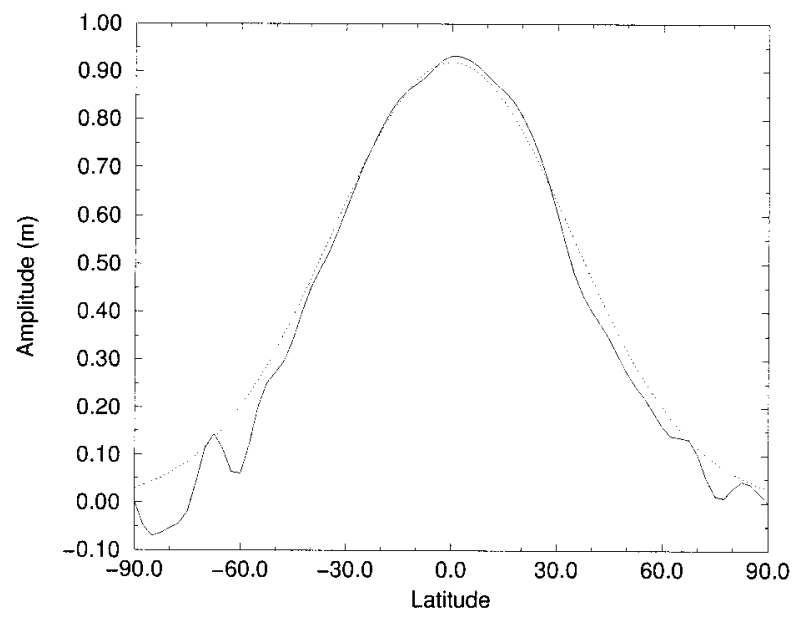

FIG. 11. As in Fig. 8 but for a meridional section of the zonalwavenumber- 1 component of the 500-hPa geopotential height anomaly through $25^{\circ} \mathrm{W}$ (solid line). The meridional structure of a theoretical equatorial Kelvin wave with $c=340 \mathrm{~m} \mathrm{~s}^{-1}$ is shown by the dotted line.

zonal-wavenumber-1 component of tropical OLR (averaged from $25^{\circ} \mathrm{S}$ to $25^{\circ} \mathrm{N}$ ) was constructed. This showed a pronounced annual cycle, which can be contrasted with the lack of an annual cycle in the 33-h Kelvin wave as discussed above. A cospectral analysis with the time series of the amplitude of the 33-h Kelvin wave was performed. If the MJO were responsible for forcing the Kelvin wave, then enhanced coherence squared would be expected between frequencies of approximately $1 / 60$ and $1 / 30$ day $^{-1}$. Although there was a large peak in the power spectrum of the OLR time series in this frequency band, no such peak in the coherence squared was found at this, or other, frequencies. Also, the overall correlation coefficient between the two time series was not distinguishable from zero at the $90 \%$ level.

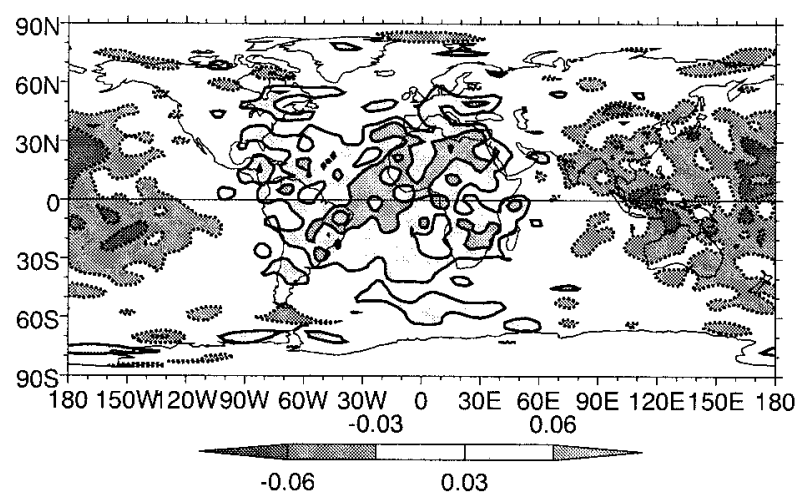

FIG. 12. As in Fig. 8 but for 10 -hPa zonal wind. Contour interval is $0.03 \mathrm{~m} \mathrm{~s}^{-1}$; negative contours are dotted and the zero contour is omitted. Shading is shown by the legend. 


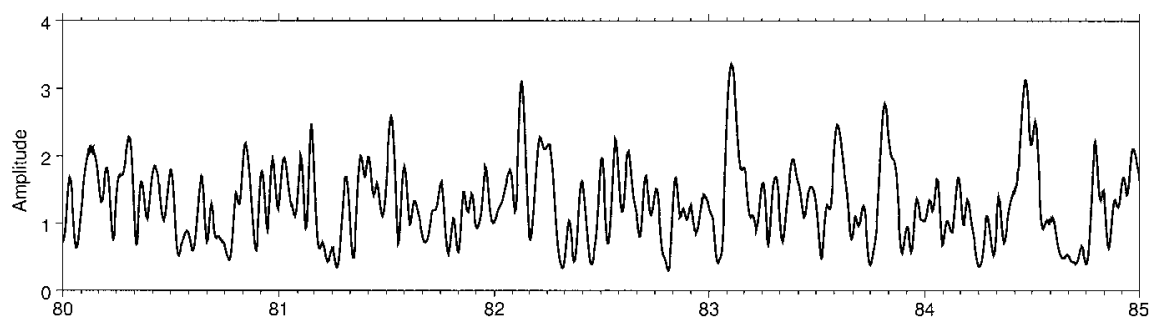

FIG. 13. Time series of the amplitude $A$ of the 33-h Kelvin wave for 1985-89. There are tick marks on the horizontal axis at the beginning of each month and the labels denote 1 Jan of that year. The data have been smoothed by an 8-day running mean.

\section{Comparison between reanalysis and station data}

The 33-h Kelvin wave has been shown to exist both in the station data (section 4) and the reanalysis data (section 5). However, as the reanalysis has a modelgenerated component in addition to its observational input, there is a possibility that the Kelvin wave in the reanalysis could arise from this model component, not the observations. Therefore, in this section cross-spectral analysis is used to search for a coherent relationship between the Kelvin wave in the two datasets.

Figure 14a shows the coherence squared between the PC1 time series from the reanalysis and the Port of Spain station pressure data. For these calculations, PC1 is the projection of the EOF1 spatial structure in Fig. 7a onto the unfiltered gridpoint maps of SLP so that it poten- tially contains variance at all frequencies, except for the annual and diurnal cycles, which have been removed. There is a large peak in the coherence squared in the frequency range $0.69-0.79 \mathrm{cpd} .{ }^{1}$ The phase difference is such that PC1 lags Port of Spain pressure by 0.6 radians or $34^{\circ}$ of phase over this frequency range (Fig. 14b). There is also significant coherence squared between PC2 and Port of Spain pressure in the 0.69-0.79cpd frequency range (Fig. 14c) with PC2 lagging Port

\footnotetext{
${ }^{1}$ There is also a large peak near $0.2 \mathrm{cpd}$. This corresponds to the westward-propagating 5-day wave (Madden and Julian 1972), which also has a zonal-wavenumber-1 structure and large amplitude at the equator.
}
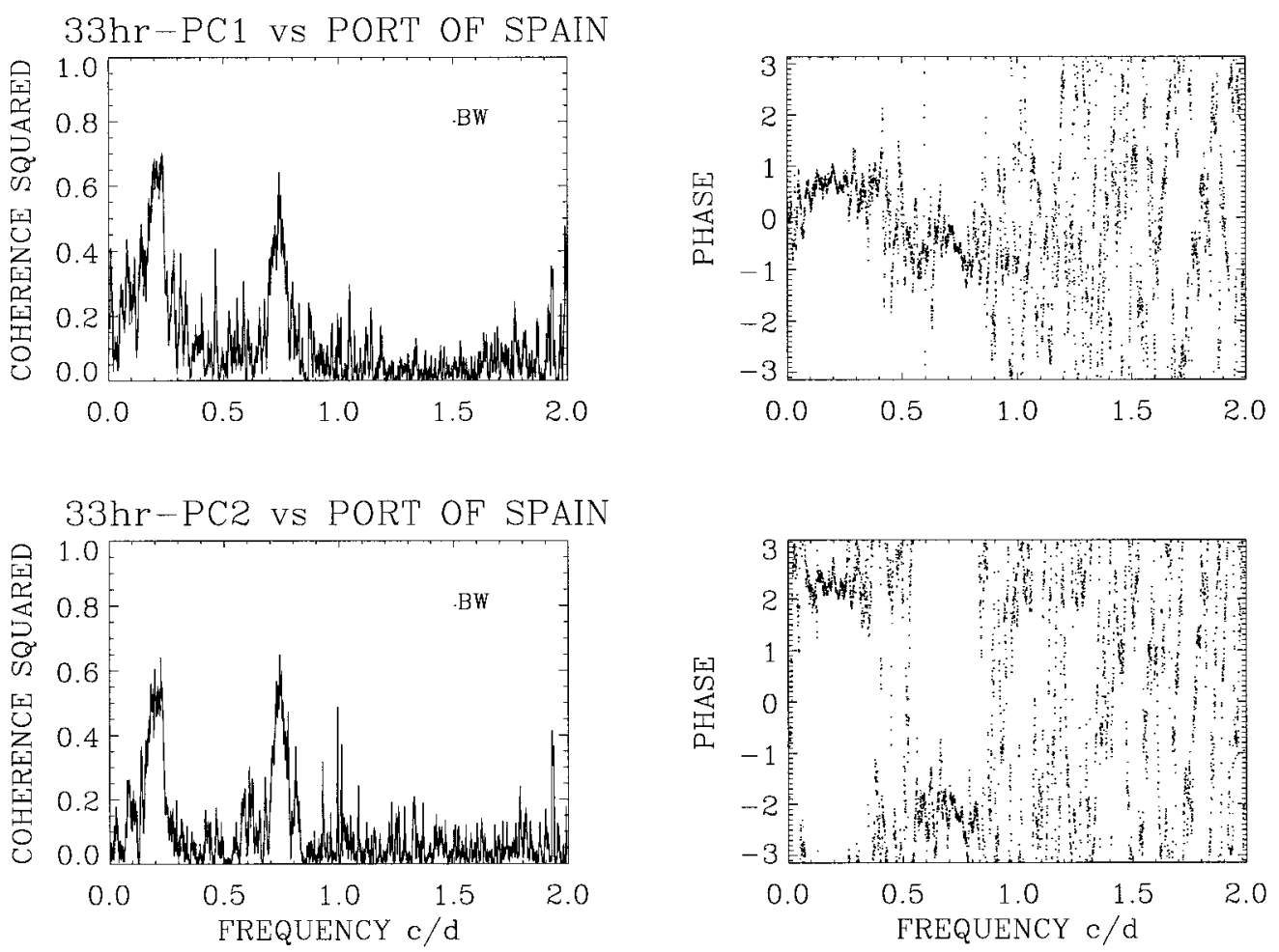

FIG. 14. Cross-spectral analysis between PC1 and Port of Spain pressure time series. (a) Coherence squared,

(b) phase difference (radians). (c), (d) as for (a) and (b) but between PC2 and Port of Spain. 
of Spain by approximately 2.2 radians or $126^{\circ}$ (Fig. $14 \mathrm{~d}$ ), consistent with PC1 leading PC2 by approximately a quarter of a cycle.

The 33-h Kelvin wave has a zonal-wavenumber-1 structure. Therefore, as PC1 lags Port of Spain station pressure by $34^{\circ}$ of phase, then the maximum in the spatial structure of EOF1 should be $34^{\circ}$ longitude to the east of Port of Spain. The spatial structure of EOF1 has a maximum at approximately $25^{\circ} \mathrm{W}$ (Fig. 7a) and Port of Spain is at $61^{\circ} \mathrm{W}$ (Fig. 1), a difference of $36^{\circ}$ longitude. From these results it can be concluded that the 33-h Kelvin wave seen in the reanalysis data is real and not just a model-generated artifact.

\section{Discussion}

The structure of the 33-h atmospheric Kelvin wave has been analyzed using 6 years of tropical station pressure data and 31 years of global NCEP-NCAR reanalysis data. It is a zonal-wavenumber-1 eastward-propagating wave with a phase speed of approximately 340 $\mathrm{m} \mathrm{s}^{-1}$ and an equatorial trapping scale of approximately $34^{\circ}$ lat. The vertical structure has no phase change with height. The amplitude of the wave is approximately constant throughout the troposphere, with an equatorial geopotential height perturbation of $0.9 \mathrm{~m}$, then increases as $\left(p / p_{s}\right)^{-\kappa}$ in the stratosphere.

All aspects of the structure described above agree with that of the theoretically expected Kelvin wave external normal mode, except for the constant amplitude in the troposphere. The reason for this discrepancy is unclear, although the assumption of an isothermal atmosphere in the theoretical treatment may be a factor.

Given the small amplitude of the wave (an SLP perturbation of $A \approx 10 \mathrm{~Pa}$ ), the close agreement between the station and reanalysis data is worth emphasising. In particular, the amplitude and phase of the wave are consistent between the two datasets. The structure of the 33-h Kelvin wave could be used to validate the data assimilation and analysis schemes.

The forcing mechanism for the wave is still not understood, and we found no preferred 33-h timescale in deep tropical convection. However, random stochastic forcing will project onto all the atmospheric normal modes (Salby and Garcia 1987), including the external-mode Kelvin wave structure. Hence, although the vertical structure of tropical convection is such that it will project only slightly onto the external mode, random convective forcing could still excite the 33-h Kelvin wave with a low amplitude. This scenario could be investigated by forcing an atmospheric model with random convection having the same temporal and spatial covariance statistics as observed convection, following Salby and Garcia. Stochastic forcing by other mechanisms, such as dry atmospheric disturbances, may be important. It is important to note that the small amplitude of the wave relative to other atmospheric variability may make it difficult to identify a single or dominant forcing mechanism.
Acknowledgments. The authors thank David Karoly for helpful discussions and comments, and RAM thanks J. Caron and T. Kestin for help with IDL. The comments of two anonymous reviewers helped to improve the manuscript from its original version. The station data were provided by Will Spangler of the Data Support Section at NCAR (http://www.scd.ucar.edu/dss/). The NOAA Climate Diagnostics Center (http:// www.cdc.noaa.gov) provided the NCEP-NCAR reanalysis data and, together with Dr. Matthew Wheeler, the OLR data. This research was carried out while one of the authors (RAM) was a visitor at the Cooperative Research Centre for Southern Hemisphere Meteorology. This research was supported in part through the Australian Government Cooperative Research Centres Program.

\section{REFERENCES}

Andrews, D. G., J. R. Holton, and C. B. Leovy, 1987: Middle Atmosphere Dynamics. Academic Press, 489 pp.

Hamilton, K., 1984: Evidence for a normal mode Kelvin wave in the atmosphere. J. Meteor. Soc. Japan, 62, 308-311.

, and R. R. Garcia, 1986: Observations of the short-period normal mode oscillations of the atmosphere. J. Geophys. Res., 91, $11867-11875$.

Haurwitz, B., 1937: The oscillations of the atmosphere. Gerlands Beitr. Geophys., 51, 195-233.

Hough, S. S., 1898: On the application of harmonic analysis to the dynamical theory of the tides. II. On the general integration of Laplace's tidal equations. Philos. Trans. Roy. Soc. London, 191A, 139-185.

Jenkins, G. M., and D. G. Watts, 1968: Spectral Analysis and Its Applications. Holden-Day, 523 pp.

Kalnay, E., and Coauthors, 1996: The NCEP/NCAR 40-Year Reanalysis Project. Bull. Amer. Meteor. Soc., 77, 437-471.

Liebmann, B., and C. A. Smith, 1996: Description of a complete (interpolated) OLR dataset. Bull. Amer. Meteor. Soc., 77, 12751277.

Longuet-Higgins, M. S., 1968: The eigenfunctions of Laplace's tidal equations over a sphere. Philos. Trans. Roy. Soc. London, 262A, 511-607.

Madden, R. A., and P. R. Julian, 1972: Further evidence of globalscale 5-day pressure waves. J. Atmos. Sci., 29, 1464-1469.

Matsuno, T., 1966: Quasi-geostrophic motions in the equatorial area. J. Meteor. Soc. Japan, 44, 25-42.

_ 1980: A trial search for minor components of lunar tides and short period free oscillations of the atmosphere in surface pressure data. J. Meteor. Soc. Japan, 58, 281-285.

Milliff, R. F., and R. A. Madden, 1996: The existence and vertical structure of fast, eastward-moving disturbances in the equatorial troposphere. J. Atmos. Sci., 53, 586-597.

North, G. R., T. L. Bell, R. F. Cahalan, and F. J. Moeng, 1982: Sampling errors in the estimation of empirical orthogonal functions. Mon. Wea. Rev., 110, 699-706.

Salby, M. L., 1979: On the solution of the homogeneous vertical structure problem. J. Atmos. Sci., 36, 2350-2359.

— heating in the Tropics. Part I: Excitation and short-time nearfield behavior. J. Atmos. Sci., 44, 458-498.

Wheeler, M., and G. N. Kiladis, 1999: Convectively coupled equatorial waves: Analysis of clouds and temperature in the wavenumber-frequency domain. J. Atmos. Sci., 56, 374-399.

Wilks, D. S., 1995: Statistical Methods in the Atmospheric Sciences. Academic Press, 467 pp. 